\title{
Studies on Heterosis in Brinjal (Solanum melongena L.)
}

\author{
Pramila*, M.L. Kushwaha and Yamuna Prasad Singh \\ Department of Vegetable Science, G.B. Pant University of Agriculture and Technology, \\ Pantnagar-263145, Uttarakhan, India \\ *Corresponding author
}

\begin{tabular}{|c|c|}
\hline \multicolumn{2}{|r|}{ A B S T R A C T } \\
\hline & \multirow{6}{*}{ 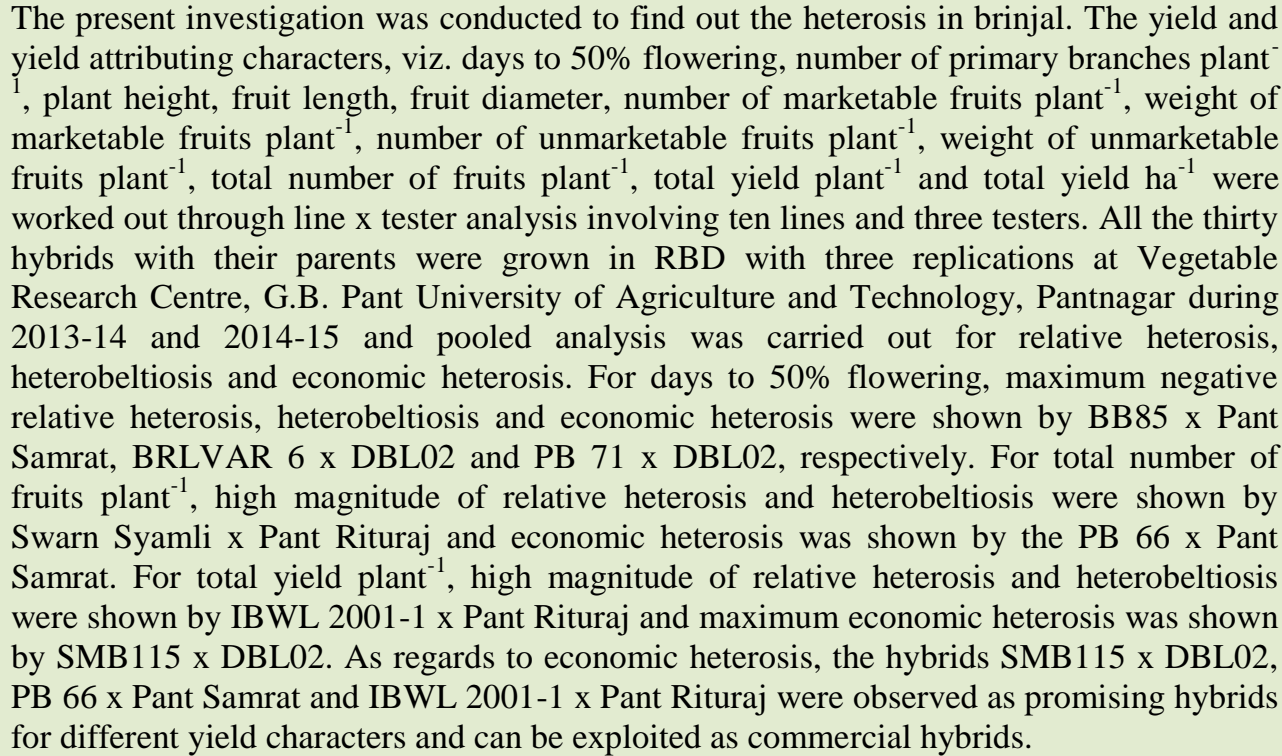 } \\
\hline & \\
\hline & \\
\hline Article Info & \\
\hline $\begin{array}{l}\text { Accepted: } \\
\text { 07 September } 2017 \\
\text { Available Online: } \\
10 \text { November } 2017\end{array}$ & \\
\hline & \\
\hline
\end{tabular}

\section{Introduction}

Brinjal (Solanum melongena L) is an important vegetable crop of India and is grown throughout the year. However, it is widely cultivated in both temperate and tropical regions of the globe mainly for its immature fruits as vegetables (Rai et al., 1995). Globally, India is the largest producer of vegetables and ranks second in production of brinjal or eggplant. However, the present production and productivity of eggplant is not enough to meet the nutritional security of increasing population. Additionally, there are also regional preferences for fruit shape, size, taste, colour etc as these traits vary significantly with the type of eggplant cultivar. The fruits of eggplant are widely consumed in various culinary preparations and are rich source of protective nutrients (Hedges and Lister, 2007). Eggplant contains a higher content of free reducing sugars, anthocyanin, phenols, glycoalkaloids (solasodine) and amide proteins. Bitterness in eggplant is due to the presence of saponins and glycoalkaloids (Mariola et al., 2013). 
Eggplant is well known for its medicinal properties and has also been recommended as an excellent remedy for liver complaints and diabetic patients (Tiwari et al., 2009). Due to the multiple health benefits of eggplant, which include anti-oxidant, anti-diabetic, hypotensive, cardioprotective and hepatoprotective effects, the demand for eggplant has been on a rapid and steady rise in the recent years (Ojiewo et al., 2007). Heterosis breeding has become the widely used breeding method for increasing productivity of the important solanaceous vegetable crops including brinjal. Ease of handling the flowers during artificial emasculation and pollination and realization of higher number of hybrid seeds per effective pollination causes higher yield of hybrids. Lack of appropriate hybrids for specific area and purpose is the major problem in popularizing the hybrids of brinjal. In the present investigation, thirteen parents were selected on the basis of divergence. They were mated in line $\times$ tester mating design to raise hybrids and relative heterosis, heterobeltiosis and standard heterosis was measured for different yield attributes.

\section{Materials and Methods}

Field experiment was conducted at Vegetable Research Centre of G. B. Pant University of Agriculture and Technology, Pantnagar, Udham Singh Nagar (Uttarakhand), India. The University lies in south of the Shivalik range of Himalayas. It falls in humid subtropical zone locally known as 'Tarai' situated at latitude of $29^{\circ}$ North, longitude of $79.3^{0}$ East and altitude of 243.84 meters above mean sea level (MSL).The materials for the present investigation comprised of 13 genotypes of Brinjal including 10 lines and 3 testers from germplasm maintained at Vegetable Research Centre. The 13 parental lines were agronomically and morphologically diverse. The genotypes were
Swarn Syamli, PB71, BARI, SMB115, BB85, BRLVAR6, IBWL2001-1, PB66, LC7, PB70, Pant Samrat, Pant Rituraj and DBL-02. Out of thirteen genotypes, the ten were used as female (lines), viz. Swarn Syamli,PB71, BARI, SMB115, BB85, BRLVAR6, IBWL2001-1, PB66, LC7, PB70 and three as male (testers), viz. Pant Samrat, Pant Rituraj DBL-02. Crossing was made in line $\times$ tester mating design statistical analysis was done as per Kempthorne (1957) model to evaluate the 30 hybrids along with 13 parents for different yield attributes. Hybrids were evaluated for two successive years in 20013-14 and 201415 autumn-winter season and pooled analysis was carried out. The layout of the experiment was Randomized Block Design with three replications. Each plot consisted of 10 plants planted at a distance of $75 \mathrm{~cm} \times 60 \mathrm{~cm}$. Data were taken from randomly selected 5 plants from each replication. Pooled magnitude of heterosis over mid-parent (MP), better-parent (BP) and standard parent (SP) were calculated.

\section{Results and Discussion}

Pooled data of two years indicated that coefficient of variation (CV) was in the range of 5.42 to 24.52 (Table 1). This indicated that the precision of experiment was within the accepted normal limit of coefficient of variation. The general means for days to $50 \%$ flowering was 69.95 and range for the same was 60.67 (in parent DBL02 and cross PB71 $\times$ DBL02) to 84.00 (BRLVAR6). The number of primary branches per plant showed the range of 2.20 (PB71) to $5.33(\mathrm{~PB} 70 \times$ DBL02) with general mean 3.52. For plant height general mean was 80.99 and mean value ranges from 61.43 (DBL02) to 92.54 (PB70 $\times$ Pant Rituraj). General mean of 15.37 and it ranged from 7.00 (Swarn Syamli) to $24.49($ BARI $\times$ DBL02) for fruit length and for fruit diameter the range 29.83 (IBWL2001-1) to 83.82 (PB70) with general 
mean 50.24. General mean for number of marketable and unmarketable fruits per plant was 17.19 and 4.75 , respectively. It ranged from 8.04 (Swarn Syamli) to 37.41 (PB66 $\times$ Pant Samrat) for number of marketable fruits per plant and 2.32 (PB $70 \times$ DBL02) to 11.09 (PB66 $\times$ Pant Samrat) for number of unmarketable fruits per plant. General mean for weight of marketable and unmarketable fruits per plant were 1.46 and $0.48 \mathrm{~kg}$, respectively. Whereas, the range of mean value for weight of marketable fruits per plant was $0.79 \mathrm{Kg}$ (BB85) to $2.49 \mathrm{Kg}$ (SMB115 × DBL02) and for weight of unmarketable fruits per plant was $0.12 \mathrm{Kg}$ (Swarn Syamli $\times$ pant Samrat) to $0.87 \mathrm{Kg}$ (IBWL2001-1 $\times$ Pant Rituraj). For total number of fruits per plant general mean 21.94 and ranged from 10.63 (Swarn Syamli) to 48.49 (PB66 $\times$ Pant Samrat) and for total yield per plant general mean 1.94 and it ranged from $0.98 \mathrm{Kg}$ (BB85) to $3.15 \mathrm{Kg}(\mathrm{SMB} 115 \times \mathrm{DBL} 02)$. General mean 430.17 and it ranged 216.10 (BB85) to $700.91($ SMB115 $\times$ DBL02) for total yield per hectare.

Wide range of variability existed among parents and their $F_{1}$ hybrids for different traits under study. Table 2, 3 and 4 represent per cent heterosis over mid, better and standard parent, respectively. Out of the 30 hybrids, the significant desirable heterotic effects over their respective mid, better and standard parent were noticed in 16, 19 and 21 crosses for days to $50 \%$ flowering, 23, 22 and 18 crosses for primary branches per plant, 18, 16 and 11 crosses for plant height, 18, 21 and 25 crosses for fruit length, 19, 21 and 24 crosses for fruit diameter, 27, 18 and 20 crosses for number of marketable fruits per plant, 26, 24 and 22 crosses for weight of marketable fruits per plant, 28, 29 and 28 crosses for number of unmarketable fruits per plant, 30, 28 and 28 crosses for weight of unmarketable fruits per plant, 22, 20 and 20 crosses for total number of fruits per plant, 26, 26 and 24 crosses for total yield per plant and 27, 26 and 26 for total yield/ hectare. The best significant hybrids for different traits with respect to heterosis over mid parent, better parent and check variety are presented in Table 5.

In case of days to $50 \%$ flowering where negative heterosis is desirable 7,19 and 5 crosses showed negative significant relative, heterobeltiosis and economic heterosis, respectively. Maximum negative relative heterosis was observed in cross BB85 $\times$ Pant Samrat (-7.43\%), maximum negative heterobeltiosis in BRLVAR6 $\times$ DBL02 $(-$ $19.44 \%$ ) and maximum negative economic heterosis was recorded in PB71 $\times$ DBL02 ($7.85 \%$ ), which was in conformity with the study of Singh et al.,(2002), Ansari et al., (2009), Das et al., (2009) and Biswas et al., (2013).

For number of primary branches per plant 12,7 and 16 crosses showed positive significant relative heterosis, heterobeltiosis and economic heterosis, respectively. Best cross with positive relative heterosis was BRLVAR6 $\times$ Pant Samrat $(81.48 \%)$, again BRLVAR6 $\times$ Pant Samrat $(56.38 \%)$ was best for heterobeltiosis, best cross for economic heterosis was PB $70 \times$ DBL02 (70.21\%). These findings are similar with the results of Das et al., (2009) and Dubey et al., (2014).

The results on heterosis for plant height indicated existence of positive significant relative heterosis, heterobeltiosis and economic heterosis 18,10 and 10 crosses, respectively. Maximum positive relative heterosis was observed in cross Swarn Syamli $\times$ Pant Rituraj (23.22\%) again maximum heterobetiosis was shown by the cross Swarn Syamli $\times$ Pant Rituraj (19.15\%) and maximum economic heterosis was observed in PB70 × Pant Samrat (16.50\%). Das et al., (2009) and Biswas et al., (2013) reported similar results. 
Table.1 Mean performance of hybrids and their parents for twelve characters

\begin{tabular}{|c|c|c|c|c|c|c|c|c|c|c|c|c|}
\hline Genotypes & $\begin{array}{c}\text { Days to } \\
50 \% \\
\text { flowering }\end{array}$ & $\begin{array}{l}\text { No. of } \\
\text { primary } \\
\text { branches } \\
\text { plant }^{-1}\end{array}$ & $\begin{array}{l}\text { Plant } \\
\text { height } \\
(\mathrm{cm})\end{array}$ & $\begin{array}{c}\text { Fruit } \\
\text { length } \\
(\mathrm{cm})\end{array}$ & $\begin{array}{c}\text { Fruit } \\
\text { diameter } \\
(\mathrm{mm})\end{array}$ & $\begin{array}{c}\text { No. of } \\
\text { marketable } \\
\text { fruits plant } \\
{ }_{1}^{-}\end{array}$ & $\begin{array}{c}\text { No. of } \\
\text { unmarketable } \\
\text { fruits plant }^{-1}\end{array}$ & $\begin{array}{c}\text { Wt. of } \\
\text { marketable } \\
\text { fruits plant } \\
{ }^{1}(\mathrm{~kg})\end{array}$ & $\begin{array}{c}\text { Wt. of } \\
\text { unmarketable } \\
\text { fruits plant }^{-1} \\
(\mathrm{~kg})\end{array}$ & $\begin{array}{l}\text { Total } \\
\text { no. of } \\
\text { fruits } \\
\text { plant }^{-1}\end{array}$ & $\begin{array}{c}\text { Total } \\
\text { yield } \\
\text { plant }^{-1} \\
(\mathrm{~kg})\end{array}$ & $\begin{array}{c}\text { Total } \\
\text { yield } \\
\text { hectare } \\
\text { (q) }\end{array}$ \\
\hline Swarn SyamlixPant Samrat & 69.50 & 4.36 & 85.76 & 12.85 & 37.76 & 28.81 & 2.75 & 0.97 & 0.12 & 31.55 & 1.09 & 242.03 \\
\hline $\begin{array}{l}\text { Swarn Syamli XPant } \\
\text { Rituraj }\end{array}$ & 73.66 & 3.13 & 86.66 & 11.34 & 76.64 & 17.75 & 3.69 & 1.53 & 0.41 & 21.44 & 1.94 & 432.02 \\
\hline Swarn Syamli xDBL 02 & 64.83 & 2.73 & 75.20 & 14.65 & 49.02 & 17.96 & 7.77 & 1.61 & 0.67 & 25.74 & 2.29 & 508.44 \\
\hline PB 71xPant Samrat & 64.16 & 4.40 & 92.43 & 15.24 & 41.72 & 22.97 & 4.74 & 1.54 & 0.55 & 27.71 & 2.10 & 466.28 \\
\hline PB 71xPant Rituraj & 66.66 & 3.83 & 90.30 & 12.31 & 57.86 & 19.74 & 4.38 & 1.72 & 0.61 & 24.11 & 2.33 & 518.05 \\
\hline PB 71xDBL 02 & 60.66 & 3.53 & 76.90 & 19.55 & 43.54 & 9.54 & 3.66 & 0.94 & 0.34 & 13.20 & 1.28 & 284.45 \\
\hline BARIxPant Samrat & 74.00 & 3.46 & 80.96 & 18.69 & 41.08 & 19.03 & 5.22 & 1.16 & 0.39 & 24.25 & 1.55 & 344.21 \\
\hline BARIXPant Rituraj & 79.16 & 3.13 & 83.40 & 19.51 & 41.14 & 13.97 & 4.60 & 1.32 & 0.43 & 18.56 & 1.75 & 389.32 \\
\hline BARIXDBL 02 & 74.00 & 3.30 & 82.33 & 24.49 & 33.44 & 16.61 & 6.02 & 1.28 & 0.39 & 22.63 & 1.67 & 370.34 \\
\hline SMB $115 \times$ Pant Samrat & 71.16 & 3.96 & 87.63 & 14.63 & 34.22 & 20.75 & 6.93 & 1.55 & 0.59 & 27.67 & 2.14 & 475.70 \\
\hline SMB 115xPant Rituraj & 74.00 & 3.70 & 76.33 & 10.76 & 62.91 & 20.51 & 4.13 & 1.57 & 0.45 & 24.64 & 2.01 & 447.65 \\
\hline SMB $115 \times D B L 02$ & 68.16 & 2.66 & 77.80 & 18.08 & 39.71 & 32.14 & 7.23 & 2.49 & 0.66 & 39.37 & 3.15 & 700.91 \\
\hline BB $85 \times$ Pant Samrat & 62.33 & 3.26 & 78.23 & 14.42 & 41.93 & 27.88 & 6.61 & 1.51 & 0.44 & 34.49 & 1.95 & 433.50 \\
\hline BB 85xPant Rituraj & 69.16 & 2.83 & 84.93 & 11.46 & 66.77 & 15.27 & 5.17 & 1.49 & 0.52 & 20.44 & 2.01 & 447.25 \\
\hline BB $85 \times$ DBL 02 & 62.16 & 3.56 & 82.93 & 17.05 & 42.23 & 22.65 & 5.67 & 1.54 & 0.53 & 28.32 & 2.07 & 459.10 \\
\hline BRLVAR 6xPant Samrat & 76.00 & 4.90 & 86.43 & 17.16 & 44.92 & 20.24 & 4.69 & 1.68 & 0.48 & 24.93 & 2.16 & 480.99 \\
\hline BRLVAR 6xPant Rituraj & 79.66 & 4.40 & 92.53 & 14.51 & 70.77 & 16.53 & 4.56 & 2.15 & 0.71 & 21.09 & 2.86 & 635.80 \\
\hline BRLVAR $6 \times$ DBL 02 & 67.66 & 4.20 & 84.56 & 14.90 & 31.21 & 15.41 & 4.99 & 1.38 & 0.48 & 20.40 & 1.86 & 413.56 \\
\hline IBWL 2001-1xPant Samrat & 62.83 & 3.03 & 86.26 & 15.77 & 31.20 & 21.88 & 7.02 & 1.43 & 0.48 & 28.91 & 1.91 & 424.96 \\
\hline IBWL 2001-1xPant Rituraj & 68.50 & 2.56 & 82.53 & 16.13 & 67.48 & 18.42 & 4.90 & 2.06 & 0.87 & 23.31 & 2.93 & 652.06 \\
\hline IBWL 2001-1XDBL 02 & 64.83 & 2.93 & 76.96 & 21.12 & 33.25 & 14.97 & 5.61 & 1.17 & 0.52 & 20.58 & 1.68 & 374.01 \\
\hline PB 66xPant Samrat & 64.00 & 4.03 & 80.46 & 19.62 & 39.04 & 37.41 & 11.09 & 2.29 & 0.77 & 48.49 & 3.06 & 680.54 \\
\hline PB $66 \times$ Pant Rituraj & 67.50 & 3.16 & 80.03 & 17.09 & 56.31 & 20.24 & 6.59 & 1.83 & 0.62 & 26.83 & 2.46 & 546.29 \\
\hline PB 66xDBL 02 & 63.16 & 3.60 & 74.46 & 17.77 & 47.61 & 14.64 & 5.31 & 1.75 & 0.75 & 19.95 & 2.50 & 555.77 \\
\hline
\end{tabular}


Conti....

\begin{tabular}{|c|c|c|c|c|c|c|c|c|c|c|c|c|}
\hline Genotypes & $\begin{array}{c}\text { Days to } \\
50 \% \\
\text { flowering }\end{array}$ & $\begin{array}{c}\text { No. of } \\
\text { primary } \\
\text { branches/plant }\end{array}$ & $\begin{array}{l}\text { Plant } \\
\text { height } \\
(\mathrm{cm})\end{array}$ & $\begin{array}{l}\text { Fruit } \\
\text { length } \\
(\mathrm{cm})\end{array}$ & $\begin{array}{c}\text { Fruit } \\
\text { diameter } \\
(\mathrm{mm})\end{array}$ & $\begin{array}{c}\text { No. of } \\
\text { marketable } \\
\text { fruits plant } \\
\end{array}$ & $\begin{array}{c}\text { No. of } \\
\text { unmarketable } \\
\text { fruits plant }^{-1}\end{array}$ & $\begin{array}{c}\text { Wt. of } \\
\text { marketable } \\
\text { fruits plant } \\
{ }^{1}(\mathrm{~kg})\end{array}$ & $\begin{array}{c}\text { Wt. of } \\
\text { unmarketable } \\
\text { fruits plant }^{-1} \\
(\mathrm{~kg})\end{array}$ & $\begin{array}{l}\text { Total no. } \\
\text { of fruits } \\
\text { plant }^{-1}\end{array}$ & $\begin{array}{c}\text { Total } \\
\text { yield } \\
\text { plant }^{-1} \\
(\mathrm{~kg})\end{array}$ & $\begin{array}{l}\text { Total } \\
\text { yield } \\
\text { hectare } \\
{ }^{1} \text { (q) }\end{array}$ \\
\hline LC $7 \times$ Pant Samrat & 71.17 & 3.90 & 73.30 & 16.16 & 51.37 & 16.37 & 3.28 & 1.78 & 0.40 & 19.65 & 2.18 & 483.67 \\
\hline LC 7XPant Rituraj & 78.00 & 2.90 & 78.70 & 12.10 & 67.48 & 13.09 & 2.98 & 1.96 & 0.52 & 16.07 & 2.48 & 550.59 \\
\hline LC 7XDBL 02 & 72.17 & 3.77 & 78.43 & 17.87 & 50.16 & 10.18 & 2.39 & 1.67 & 0.47 & 12.56 & 2.14 & 475.89 \\
\hline PB 70xPant Samrat & 69.00 & 3.60 & 86.33 & 20.12 & 42.24 & 12.16 & 2.80 & 1.32 & 0.33 & 14.96 & 1.64 & 365.55 \\
\hline PB 70xPant Rituraj & 77.83 & 4.10 & 92.54 & 12.82 & 73.11 & 9.15 & 3.26 & 1.73 & 0.73 & 12.41 & 2.45 & 545.36 \\
\hline PB 70xDBL 02 & 66.50 & 5.33 & 81.23 & 16.57 & 54.11 & 9.67 & 2.32 & 1.28 & 0.42 & 11.99 & 1.71 & 379.16 \\
\hline Swarn Syamli & 69.33 & 3.13 & 68.23 & 7.00 & 73.05 & 8.04 & 2.59 & 1.42 & 0.28 & 10.63 & 1.70 & 377.74 \\
\hline PB 71 & 63.50 & 2.20 & 92.07 & 12.47 & 56.61 & 14.53 & 3.34 & 1.22 & 0.32 & 17.87 & 1.54 & 342.29 \\
\hline BARI & 78.50 & 4.33 & 80.60 & 23.87 & 33.28 & 9.51 & 3.60 & 1.02 & 0.38 & 13.11 & 1.40 & 311.79 \\
\hline SMB 115 & 70.33 & 2.80 & 87.33 & 11.55 & 48.58 & 18.87 & 4.93 & 1.50 & 0.50 & 23.80 & 2.00 & 443.44 \\
\hline BB 85 & 68.83 & 4.17 & 73.70 & 11.71 & 42.93 & 15.84 & 3.99 & 0.79 & 0.19 & 19.83 & 0.98 & 217.00 \\
\hline BRLVAR 6 & 84.00 & 2.27 & 79.10 & 14.54 & 59.68 & 10.20 & 2.74 & 0.91 & 0.26 & 12.94 & 1.17 & 260.52 \\
\hline IBWL 2001-1 & 69.00 & 3.27 & 66.77 & 13.56 & 29.83 & 19.37 & 3.56 & 0.94 & 0.18 & 22.92 & 1.12 & 249.76 \\
\hline PB 66 & 65.50 & 3.77 & 81.10 & 19.55 & 43.69 & 22.58 & 7.73 & 1.45 & 0.66 & 30.31 & 2.11 & 468.66 \\
\hline LC 7 & 79.33 & 2.70 & 75.10 & 14.02 & 79.64 & 8.95 & 2.35 & 1.26 & 0.38 & 11.30 & 1.64 & 364.49 \\
\hline PB 70 & 78.17 & 3.57 & 88.68 & 14.27 & 83.82 & 10.99 & 4.86 & 1.62 & 0.75 & 15.85 & 2.37 & 527.13 \\
\hline Pant Samrat & 65.83 & 3.13 & 79.43 & 20.26 & 35.59 & 19.54 & 5.78 & 1.12 & 0.34 & 25.32 & 1.46 & 323.62 \\
\hline Pant Rituraj & 72.33 & 4.27 & 72.43 & 8.41 & 69.32 & 8.97 & 3.79 & 0.85 & 0.29 & 12.76 & 1.14 & 253.06 \\
\hline DBL 02 & 60.67 & 3.37 & 61.43 & 20.64 & 33.85 & 15.96 & 4.71 & 0.94 & 0.29 & 20.67 & 1.24 & 274.62 \\
\hline General Mean & 69.95 & 3.52 & 80.99 & 15.74 & 50.24 & 17.19 & 4.75 & 1.46 & 0.48 & 21.94 & 1.94 & 430.17 \\
\hline C.V. & 5.45 & 9.38 & 9.55 & 9.21 & 8.46 & 16.72 & 24.58 & 15.11 & 23.35 & 13.31 & 11.42 & 11.78 \\
\hline S.E. & 1.56 & 0.13 & 3.16 & 0.59 & 1.73 & 1.17 & 0.48 & 0.09 & 0.05 & 1.19 & 0.09 & 20.69 \\
\hline C.D. $5 \%$ & 4.34 & 0.38 & 8.81 & 1.65 & 4.83 & 3.27 & 1.33 & 0.25 & 0.13 & 3.32 & 0.25 & 57.68 \\
\hline C.D. $1 \%$ & 5.72 & 0.50 & 11.61 & 2.18 & 6.38 & 4.31 & 1.75 & 0.33 & 0.17 & 4.38 & 0.33 & 76.05 \\
\hline
\end{tabular}


Table.2 Estimation of per cent relative heterosis (over mid parent)

\begin{tabular}{|c|c|c|c|c|c|c|c|c|c|c|c|c|}
\hline Hybrids & $\begin{array}{c}\text { Days to } \\
50 \% \\
\text { flowering }\end{array}$ & $\begin{array}{c}\text { No. of } \\
\text { primary } \\
\text { branches } \\
\text { plant }^{-1}\end{array}$ & $\begin{array}{l}\text { Plant } \\
\text { height } \\
(\mathrm{cm})\end{array}$ & $\begin{array}{l}\text { Fruit } \\
\text { length } \\
(\mathrm{cm})\end{array}$ & $\begin{array}{c}\text { Fruit } \\
\text { diameter } \\
(\mathrm{mm})\end{array}$ & $\begin{array}{c}\text { No. of } \\
\text { marketable } \\
\text { fruits plant }\end{array}$ & $\begin{array}{c}\text { Wt. of } \\
\text { marketable } \\
\text { fruits plant }{ }^{-1}\end{array}$ & $\begin{array}{l}\quad \text { No. of } \\
\text { unmarketable } \\
\text { fruits plant }{ }^{-1}\end{array}$ & $\begin{array}{c}\text { Wt. of } \\
\text { unmarketable } \\
\text { fruits plant }^{-1}\end{array}$ & $\begin{array}{l}\text { Total no. } \\
\text { of fruits } \\
\text { plant }^{-1}\end{array}$ & $\begin{array}{c}\text { Total } \\
\text { yield } \\
\text { plant }^{-1} \\
(\mathrm{~kg})\end{array}$ & $\begin{array}{c}\text { Total } \\
\text { yield/hectare } \\
\text { (q) }\end{array}$ \\
\hline $\begin{array}{l}\text { Swarn Shyamli } \times \text { Pant } \\
\text { Samrat }\end{array}$ & 2.84 & $39.36^{* *}$ & $16.16^{* *}$ & -5.69 & $-30.47 * *$ & $108.90 * *$ & $-23.71 * *$ & $-34.28 * *$ & $-60.56^{* *}$ & $75.58 * *$ & $-30.97 * *$ & $-30.98 * *$ \\
\hline $\begin{array}{l}\text { Swarn Shyamli } \times \text { Pant } \\
\text { Rituraj }\end{array}$ & $4.00^{*}$ & $-15.32 * *$ & $23.22 * *$ & $47.25 * *$ & $7.67 * *$ & $108.76^{* *}$ & $35.72 * *$ & $15.54 * *$ & $41.76^{* *}$ & $83.33^{* *}$ & $36.95 * *$ & $36.97 * *$ \\
\hline Swarn Shyamli $\times$ DBL02 & -0.26 & $-15.90 * *$ & $15.99 * *$ & 6.03 & $-8.28 *$ & $49.73 * *$ & $36.94 * *$ & $112.92 * *$ & $133.06^{* *}$ & $64.47 * *$ & $55.89 * *$ & $55.88 * *$ \\
\hline PB71 Pant Samrat & -0.77 & $65.00 * *$ & $7.79 *$ & -6.87 & $-9.48 *$ & $34.81 * *$ & $32.02 * *$ & 4.10 & 68.35 ** & $28.33^{* *}$ & $40.00 * *$ & $40.04 * *$ \\
\hline PB71 $\times$ Pant Rituraj & -1.84 & $18.56^{* *}$ & $9.79 * *$ & $17.98 * *$ & $-8.10 * *$ & $67.96 * *$ & $66.81 * *$ & $22.69^{* *}$ & $98.15^{* * *}$ & $57.42^{* *}$ & 73.99 & $74.03^{* *}$ \\
\hline PB71 × DBL 02 & -2.28 & $26.95 * *$ & 0.20 & $18.11^{* *}$ & -3.73 & $-37.42 * *$ & -12.69 & $-9.15^{* *}$ & $9.41 * *$ & $-31.52 * *$ & -7.80 & -7.78 \\
\hline BARI $\times$ Pant Samrat & 2.54 & -7.14 & 1.19 & $-15.29 * *$ & $19.31 * *$ & $31.04 * *$ & 8.34 & $11.34^{* *}$ & $8.40^{* *}$ & $26.23^{* *}$ & 8.35 & 8.34 \\
\hline BARI $\times$ Pant Rituraj & $4.97 * *$ & $-27.13 * *$ & $9.00 *$ & $20.90 * *$ & $-19.81 * *$ & $51.17 * *$ & $41.82 * *$ & $24.32 * *$ & $26.84 * *$ & $43.49^{* *}$ & $37.84 * *$ & $37.85 * *$ \\
\hline $\mathrm{BARI} \times \mathrm{DBL} 02$ & $6.35 * *$ & $-14.29 * *$ & $15.94 * *$ & $10.05 * *$ & -0.36 & $30.45 * *$ & $30.38 * *$ & $44.87 * *$ & $14.45 * *$ & $33.99 * *$ & $26.30 * *$ & $26.31 * *$ \\
\hline SMB115 $\times$ Pant Samrat & $4.53 * *$ & $33.71 * *$ & 5.10 & $-7.97 *$ & $-18.69 * *$ & 8.03 & $18.68 * *$ & $29.38 * *$ & $40.84 * *$ & $12.68 * *$ & $24.03 * *$ & $24.03 * *$ \\
\hline SMB115 × Pant Rituraj & $3.74 *$ & 4.72 & -4.44 & 7.89 & $6.71 *$ & $47.37 * *$ & $33.57 * *$ & $-5.43 *$ & $13.49 * *$ & $34.78^{* *}$ & $28.52 * *$ & $28.54 * *$ \\
\hline SMB115 $\times$ DBL02 & $4.07 *$ & $-13.51^{*}$ & 4.59 & $12.38 * *$ & -3.66 & $84.59 * *$ & $104.10 * *$ & $49.94 * *$ & $67.53 * *$ & $77.08^{* *}$ & $95.16^{* *}$ & $95.22 * *$ \\
\hline BB85 $\times$ Pant Samrat & $-7.43 * *$ & $-10.50 *$ & 2.18 & $-9.78 *$ & 6.80 & $57.60 * *$ & $58.61 * *$ & $35.36^{* *}$ & $66.74 * *$ & $52.79^{* *}$ & $60.36^{* *}$ & $60.37 * *$ \\
\hline BB85 $\times$ Pant Rituraj & -2.01 & $-32.81 * *$ & $16.24 * *$ & $14.02 *$ & $18.97 * *$ & $23.13 * *$ & $82.28 * *$ & $32.78 * *$ & $117.44 * *$ & $25.44 * *$ & $90.25^{* *}$ & $90.30 * *$ \\
\hline BB85 $\times$ DBL02 & $-3.99 *$ & -5.31 & $22.74 * *$ & 5.46 & $10.00^{*}$ & $42.48 * *$ & 77.59 ** & $30.37 * *$ & $119.83^{* *}$ & $39.88^{* *}$ & $86.77 * *$ & $86.77 * *$ \\
\hline BRLVAR6 $\times$ Pant Samrat & 1.45 & $81.48 * *$ & $9.04 * *$ & -1.33 & -5.70 & $36.11 * *$ & $65.43 * *$ & $10.12^{* * *}$ & $62.27 * *$ & $30.32 * *$ & $64.71 * *$ & $64.68 * *$ \\
\hline BRLVAR6 $\times$ Pant Rituraj & 1.92 & $34.69 * *$ & $22.13 * *$ & $26.56 * *$ & $9.71 * *$ & $72.49 * *$ & $144.89 * *$ & $39.64 * *$ & $156.00 * *$ & $64.14^{* *}$ & $147.54 * *$ & $147.59 * *$ \\
\hline BRLVAR6 $\times$ DBL02 & $-6.45 * *$ & $49.11 * *$ & $20.35 * *$ & $-15.28 * *$ & $-33.26 * *$ & $17.82 *$ & $48.97 * *$ & $33.97 * *$ & $73.24 * *$ & $21.40^{* *}$ & $54.54 * *$ & 54.56 ** \\
\hline IBWL2001-1 × Pant Samrat & $-6.80 * *$ & -5.21 & $18.01 * *$ & -6.72 & -4.61 & $12.50 *$ & $39.27^{* * *}$ & $50.52 * *$ & $83.52 * *$ & $19.85^{* *}$ & $48.22 * *$ & $48.23^{* *}$ \\
\hline IBWL2001-1 × Pant Rituraj & $-3.07 *$ & $-31.86^{* *}$ & $18.58 * *$ & $46.87 * *$ & 36.11 & $30.00 * *$ & $130.84 * *$ & $33.23 * *$ & $265.79 * *$ & $30.66^{* *}$ & $159.31 * *$ & $159.36^{* *}$ \\
\hline IBWL2001-1 × DBL02 & 0.00 & $-11.56^{*}$ & $20.07 * *$ & $23.52 * *$ & 4.41 & $-15.26^{*}$ & $24.04 *$ & $35.73 * *$ & $115.81 * *$ & -5.59 & $42.65^{* *}$ & $42.65 * *$ \\
\hline PB66 $\times$ Pant Samrat & -2.54 & $16.91 * *$ & 0.25 & -1.39 & -1.52 & $77.60 * *$ & $78.71 * *$ & $64.21 * *$ & $54.09 * *$ & $74.35^{* *}$ & $71.79 * *$ & $71.79 * *$ \\
\hline PB66 $\times$ Pant Rituraj & -2.06 & $-21.16 * *$ & 4.26 & $22.33 * *$ & -0.36 & $28.27 * *$ & $60.11 * *$ & $14.38 * *$ & $30.45 * *$ & $24.55^{* *}$ & $51.38 * *$ & $51.39 * *$ \\
\hline PB66 × DBL02 & 0.13 & 0.93 & 4.49 & $-11.52 * *$ & $22.80^{* *}$ & $-24.02 * *$ & $46.72 * *$ & $-14.67 * *$ & $56.60 * *$ & $-21.74 * *$ & $49.55^{* *}$ & $49.55^{* *}$ \\
\hline LC7 $\times$ Pant Samrat & -1.95 & $33.71^{* *}$ & -5.13 & -5.71 & $-10.83 * *$ & 14.90 & $49.37 * *$ & $-19.35 * *$ & $11.31 * *$ & 7.31 & $40.59 * *$ & $40.58 * *$ \\
\hline LC7× Pant Rituraj & $2.86^{*}$ & $-16.75^{* *}$ & 6.69 & 7.94 & $-9.40 * *$ & $46.12 * *$ & $85.91 * *$ & -2.99 & $54.33 * *$ & $33.59^{* *}$ & $78.30^{* *}$ & $78.31 * *$ \\
\hline LC7 $\times$ DBL02 & $3.10^{*}$ & $24.18^{* *}$ & $14.89 * *$ & 3.10 & $-11.61 * *$ & $-18.28 *$ & $51.70 * *$ & $-32.42 * *$ & $39.91 * *$ & $-21.40 * *$ & $48.95^{* *}$ & $48.92 * *$ \\
\hline PB70× Pant Samrat & $-4.17 * *$ & 7.46 & 2.71 & $16.55^{* *}$ & $-29.26 * *$ & $-20.32 * *$ & -3.84 & $-47.45 * *$ & $-39.79 * *$ & $-27.33 * *$ & $-14.07 * *$ & $-14.06^{* * *}$ \\
\hline PB70× Pant Rituraj & $3.43^{*}$ & 4.68 & $14.87 * *$ & $13.10^{*}$ & -4.52 & -8.29 & $40.03^{* *}$ & $-24.64 * *$ & $39.20 * *$ & -13.24 & $39.78^{* *}$ & $39.80^{* *}$ \\
\hline $\mathrm{PB} 70 \times \mathrm{DBL} 02$ & $-4.20 * *$ & $53.85 * *$ & $8.23 *$ & -5.08 & $-8.02 *$ & $-28.22 * *$ & 0.07 & $-51.47 * *$ & $-18.81 * *$ & $-34.32 * *$ & -5.41 & -5.42 \\
\hline
\end{tabular}


Table.3 Estimation of per cent heterobeltiosis (over better parent)

\begin{tabular}{|c|c|c|c|c|c|c|c|c|c|c|c|c|}
\hline Hybrids & $\begin{array}{c}\text { Days to } \\
50 \% \\
\text { flowering }\end{array}$ & $\begin{array}{c}\text { No. of } \\
\text { primary } \\
\text { branches } \\
\text { plant }^{-1}\end{array}$ & $\begin{array}{l}\text { Plant } \\
\text { height } \\
(\mathrm{cm})\end{array}$ & $\begin{array}{c}\text { Fruit } \\
\text { length } \\
(\mathrm{cm})\end{array}$ & $\begin{array}{c}\text { Fruit } \\
\text { diameter } \\
(\mathrm{mm})\end{array}$ & $\begin{array}{c}\text { No. of } \\
\text { marketable } \\
\text { fruits plant }^{-1}\end{array}$ & $\begin{array}{c}\text { Wt. of } \\
\text { marketable } \\
\text { fruits plant }{ }^{-1}\end{array}$ & $\begin{array}{c}\text { No. of } \\
\text { unmarketable } \\
\text { fruits plant }^{-1}\end{array}$ & $\begin{array}{c}\text { Wt. of } \\
\text { unmarketable } \\
\text { fruits plant }^{-1}\end{array}$ & $\begin{array}{l}\text { Total no. of } \\
\text { fruits plant } \\
1\end{array}$ & $\begin{array}{c}\text { Total } \\
\text { yield plant } \\
{ }^{1}(\mathrm{~kg})\end{array}$ & $\begin{array}{l}\text { Total } \\
\text { yield/hectare } \\
\text { (q) }\end{array}$ \\
\hline Swarn Syamli $\times$ Pant Samrat & 0.24 & $39.36^{* * *}$ & $7.97 *$ & $-36.54 * *$ & $-48.30 * *$ & $47.42 * *$ & $-31.72 * *$ & $-52.41 * *$ & $-63.69 * *$ & $24.64 * *$ & $-35.92 * *$ & $-35.93 * *$ \\
\hline Swarn Syamli $\times$ Pant Rituraj & 1.84 & $-26.56^{* * *}$ & $19.65 * *$ & $34.95 * *$ & 4.92 & $97.94 * *$ & 8.41 & -2.81 & $39.55^{* * *}$ & $67.99 * *$ & $14.36^{*}$ & $14.37 *$ \\
\hline Swarn Syamli $\times$ DBL02 & $-6.49 * *$ & $-18.81 * *$ & $10.21 *$ & $-28.99 * *$ & $-32.89 * *$ & 12.58 & 14.00 & $64.95 * *$ & $128.98 * *$ & $24.51 * *$ & $34.61 * *$ & 34.60 ** \\
\hline PB71 Pant Samrat & -2.53 & $40.43 * *$ & 0.40 & $-24.77 * *$ & $-26.29 * *$ & $17.54 * *$ & $26.53 * *$ & $-17.86^{* *}$ & $63.99 * *$ & 9.46 & $36.18^{* * *}$ & $36.23 * *$ \\
\hline PB71 $\times$ Pant Rituraj & $-7.83^{* *}$ & $-10.16^{*}$ & -1.92 & -1.24 & $-16.53 * *$ & $35.80^{* *}$ & $41.23^{* *}$ & $15.33^{* * *}$ & $89.80^{* * *}$ & $34.92 * *$ & $51.33^{* *}$ & $51.35^{* * *}$ \\
\hline PB71 $\times$ DBL 02 & $-4.46 *$ & 4.95 & $-16.47 * *$ & -5.26 & $-23.08 * *$ & $-40.21 * *$ & $-22.66 * *$ & $-22.39 * *$ & $4.99 *$ & $-36.15^{* *}$ & $-16.91 *$ & $-16.90 *$ \\
\hline BARI $\times$ Pant Samrat & $-5.73 * *$ & $-20.00 * *$ & 0.45 & $-21.70 * *$ & $15.44 *$ & -2.59 & 3.61 & $-9.61 * *$ & 2.09 & -4.19 & 6.37 & 6.36 \\
\hline BARI $\times$ Pant Rituraj & 0.85 & $-27.69 * *$ & 3.47 & $-18.26 * *$ & $-40.66^{* *}$ & $46.86 * *$ & $29.67 * *$ & $21.18 * *$ & $12.07 * *$ & $41.58 * *$ & $24.87 * *$ & $24.87 * *$ \\
\hline BARI $\times$ DBL02 & $-5.73 * *$ & $-23.85 * *$ & 2.15 & 2.60 & -1.20 & 4.09 & $25.31 *$ & $27.80 * *$ & 1.31 & 9.50 & $18.77 *$ & $18.78 *$ \\
\hline SMB115 $\times$ Pant Samrat & 1.18 & $26.60 * *$ & 0.34 & $-27.76 * *$ & $-29.56 * *$ & 6.18 & 3.57 & $19.91 * *$ & $18.48^{* * *}$ & 9.31 & 7.27 & 7.27 \\
\hline SMB115 $\times$ Pant Rituraj & 2.30 & $-13.28 * *$ & $-12.60 * *$ & -6.77 & $-9.26 * *$ & 8.71 & 4.43 & $-16.33^{* *}$ & $-9.63^{* * *}$ & 3.52 & 0.94 & 0.95 \\
\hline SMB115 $\times$ DBL02 & -3.08 & $-20.79 * *$ & $-10.92 * *$ & $-12.38 * *$ & $-18.27 * *$ & $70.34 * *$ & $66.06^{* * *}$ & $46.62 * *$ & 33.60 ** & $65.43^{* *}$ & $58.01 * *$ & $58.06 * *$ \\
\hline BB85 $\times$ Pant Samrat & $-9.44 * *$ & $-21.60 * *$ & -1.51 & $-28.82 * *$ & -2.33 & $42.66^{* *}$ & $35.34 * *$ & $14.46^{* *}$ & $29.35^{* * *}$ & $36.23^{* *}$ & $33.95^{* *}$ & $33.96 * *$ \\
\hline BB85 $\times$ Pant Rituraj & $-4.38 *$ & $-33.59 * *$ & $15.24 * *$ & -2.05 & -3.68 & -3.57 & $76.30^{* * *}$ & $29.48 * *$ & $77.78 * *$ & 3.08 & $76.68 * *$ & $76.74 * *$ \\
\hline BB85 $\times$ DBL02 & $-9.69 * *$ & $-14.40 * *$ & $12.53 * *$ & $-17.36 * *$ & -1.63 & $41.95^{* *}$ & 63.35 ** & $20.41 * *$ & $79.46^{* *}$ & $37.04 * *$ & $67.19^{* *}$ & $67.17 * *$ \\
\hline BRLVAR6 $\times$ Pant Samrat & $-9.52 * *$ & $56.38 * *$ & $8.81 *$ & $-15.27 * *$ & $-24.74 * *$ & 3.57 & $50.29 * *$ & $-18.82 * *$ & $43.22 * *$ & -1.53 & 48.65 ** & 48.63 ** \\
\hline BRLVAR6 $\times$ Pant Rituraj & $-5.16^{* * *}$ & 3.12 & $16.98 * *$ & -0.11 & 2.08 & $62.09 * *$ & $135.78 * *$ & $20.25 * *$ & $140.80 * *$ & $63.02 * *$ & $144.04 * *$ & $144.05^{* *}$ \\
\hline BRLVAR6 $\times$ DBL02 & $-19.44 * *$ & $24.75 * *$ & 6.91 & $-27.81 * *$ & $-47.70 * *$ & -3.44 & $46.80 * *$ & $5.94 *$ & $62.65^{* *}$ & -1.30 & $50.57 * *$ & $50.59 * *$ \\
\hline IBWL2001-1 × Pant Samrat & $-8.94 * *$ & -7.14 & $8.60^{*}$ & $-22.15 * *$ & $-12.33^{*}$ & 11.99 & $28.15^{* * *}$ & $21.59 * *$ & $41.79 * *$ & $14.18^{* *}$ & $31.31 * *$ & $31.32 * *$ \\
\hline IBWL2001-1 × Pant Rituraj & $-5.30 * *$ & $-39.84 * *$ & $13.94 * *$ & $18.98 * *$ & -2.66 & -4.90 & $119.29 * *$ & $29.04 * *$ & $197.73 * *$ & 1.71 & $157.59 * *$ & $157.67 * *$ \\
\hline IBWL2001-1 $\times$ DBL02 & $-6.04 * *$ & $-12.87 *$ & $15.28 * *$ & 2.33 & -1.79 & $-22.71 * *$ & $23.94 *$ & $19.07^{* *}$ & $75.38^{* * *}$ & -10.22 & $36.20^{* *}$ & 36.19 ** \\
\hline PB66 $\times$ Pant Samrat & -2.78 & 7.08 & -0.78 & -3.13 & $-10.65^{*}$ & $65.63^{* *}$ & 58.49 ** & $43.45^{* *}$ & $16.28^{* *}$ & $59.97 * *$ & $45.21 * *$ & $45.21 * *$ \\
\hline PB66 × Pant Rituraj & $-6.68 * *$ & $-25.78 * *$ & -1.32 & $-12.52 * *$ & $-18.78 * *$ & -10.40 & $26.90^{* *}$ & $-14.75 * *$ & $-5.93 * *$ & $-11.51 * *$ & $16.57 * *$ & $16.56^{* * *}$ \\
\hline PB66 $\times$ DBL02 & -3.56 & -4.42 & $-8.18^{*}$ & $-13.87 * *$ & 8.97 & $-35.17 * *$ & $21.13^{* * *}$ & $-31.33 * *$ & $13.07 * *$ & $-34.19 * *$ & $18.59^{* *}$ & 18.59 ** \\
\hline LC7 $\times$ Pant Samrat & $-10.29 * *$ & $24.47 * *$ & $-7.72 *$ & $-20.23 * *$ & $-35.50 * *$ & $-16.22 *$ & 40.79 ** & $-43.29 * *$ & $5.58 * *$ & $-22.40 * *$ & $32.71 * *$ & 32.70 ** \\
\hline LC7 $\times$ Pant Rituraj & -1.68 & $-32.03 * *$ & 4.79 & $-13.66 * *$ & $-15.27 * *$ & $46.00^{* *}$ & $55.17 * *$ & $-21.49 * *$ & $37.26 * *$ & $25.94 * *$ & $51.06^{* *}$ & $51.06 * *$ \\
\hline LC7 $\times$ DBL02 & $-9.03 * *$ & 11.88 & 4.44 & $-13.44 * *$ & $-37.02 * *$ & $-36.21 * *$ & $32.35 * *$ & $-49.38 * *$ & $24.65^{* * *}$ & $-39.21 * *$ & $30.59^{* *}$ & 30.56 ** \\
\hline PB70 $\times$ Pant Samrat & $-11.73 * *$ & 0.93 & -2.65 & -0.69 & $-49.61 * *$ & $-37.76 * *$ & $-18.73 * *$ & $-51.60 * *$ & $-56.36^{* *}$ & $-40.92 * *$ & $-30.66^{* *}$ & $-30.65 * *$ \\
\hline PB70 $\times$ Pant Rituraj & -0.43 & -3.91 & 4.35 & $-10.12 *$ & $-12.77 * *$ & -16.72 & 6.56 & $-32.93 * *$ & $-3.24 * *$ & $-21.69 * *$ & 3.46 & 3.46 \\
\hline PB70 $\times$ DBL02 & $-14.93 * *$ & $49.53 * *$ & $-8.40 *$ & $-19.74 * *$ & $-35.44 * *$ & $-39.40 * *$ & $-20.90 * *$ & $-52.23 * *$ & $-43.50 * *$ & $-41.97 * *$ & $-28.06 * *$ & $-28.07 * *$ \\
\hline
\end{tabular}


Table.4 Estimation of per cent economic heterosis (over check i.e. Pant Samrat)

\begin{tabular}{|c|c|c|c|c|c|c|c|c|c|c|c|c|}
\hline Hybrids & $\begin{array}{c}\text { Days to } \\
50 \% \\
\text { flowerin } \\
\mathrm{g}\end{array}$ & $\begin{array}{c}\text { No. of } \\
\text { primary } \\
\text { branches } \\
\text { plant }^{-1}\end{array}$ & $\begin{array}{l}\text { Plant } \\
\text { height } \\
(\mathrm{cm})\end{array}$ & $\begin{array}{c}\text { Fruit } \\
\text { length } \\
(\mathrm{cm})\end{array}$ & $\begin{array}{c}\text { Fruit } \\
\text { diameter } \\
(\mathrm{mm})\end{array}$ & $\begin{array}{c}\text { No. of } \\
\text { marketable } \\
\text { fruits plant }{ }^{-1}\end{array}$ & $\begin{array}{c}\text { Wt. of } \\
\text { marketable } \\
\text { fruits plant }{ }^{-1}\end{array}$ & $\begin{array}{c}\text { No. of } \\
\text { unmarketabl } \\
\mathrm{e} \\
\text { fruits plant }^{-1}\end{array}$ & $\begin{array}{c}\text { Wt.of } \\
\text { unmarketabl } \\
\text { e fruits } \\
\text { plant }^{-1}\end{array}$ & $\begin{array}{c}\text { Total no. } \\
\text { of fruits } \\
\text { plant }^{-1}\end{array}$ & $\begin{array}{l}\text { Total } \\
\text { yield } \\
\text { plant }^{-1} \\
(\mathrm{~kg})\end{array}$ & $\begin{array}{l}\text { Total yield } \\
\text { hectare }^{-1}(\mathrm{q})\end{array}$ \\
\hline Swarn Shyamli $\times$ Pant Samrat & $5.57 * *$ & $39.36^{* *}$ & $7.97 *$ & $-36.54 * *$ & 6.12 & $47.42 * *$ & -13.57 & $-52.41 * *$ & $-63.69 * *$ & $24.64 * *$ & $-25.20 * *$ & $-25.21 * *$ \\
\hline Swarn Shyamli $\times$ Pant Rituraj & $11.90 * *$ & 0.00 & $9.11^{*}$ & $-43.99 * *$ & $115.38^{* *}$ & -9.15 & $37.21^{* *}$ & $-36.16^{* *}$ & $21.16^{* *}$ & $-15.31 * *$ & $33.48 * *$ & $33.50^{* *}$ \\
\hline Swarn Shyamli $\times$ DBL02 & -1.52 & -12.77 & -5.33 & $-27.65^{* *}$ & $37.75 * *$ & -8.07 & $44.29 * *$ & $34.57 * *$ & $99.61 * *$ & 1.66 & $57.12^{* *}$ & $57.11^{* *}$ \\
\hline PB71 Pant Samrat & -2.53 & $40.43 * *$ & $16.37^{* * *}$ & $-24.77 * *$ & $17.25 * *$ & $17.54 * *$ & $38.01^{* *}$ & $-17.86^{* *}$ & $63.99 * *$ & 9.46 & $44.04 * *$ & $44.08^{* *}$ \\
\hline PB71 $\times$ Pant Rituraj & 1.27 & $22.34 * *$ & $13.68^{* *}$ & $-39.20 * *$ & $62.60 * *$ & 1.01 & $54.05^{* *}$ & $-24.24 * *$ & $79.97 * *$ & -4.75 & $60.06^{* *}$ & $60.08^{* *}$ \\
\hline PB71 $\times$ DBL 02 & $-7.85 * *$ & 12.77 & -3.19 & -3.47 & $22.35 * *$ & $-51.18 * *$ & -15.65 & $-36.68 * *$ & -0.44 & $-47.87 * *$ & -12.12 & -12.10 \\
\hline BARI $\times$ Pant Samrat & $12.41 * *$ & 10.64 & 1.93 & $-7.73^{*}$ & $15.44 *$ & -2.59 & 3.61 & $-9.61 * *$ & $15.54^{* *}$ & -4.19 & 6.37 & 6.36 \\
\hline BARI $\times$ Pant Rituraj & $20.25^{* *}$ & 0.00 & 4.99 & -3.67 & $15.60 *$ & $-28.52 * *$ & 18.34 & $-20.40 * *$ & $26.84^{* *}$ & $-26.67 * *$ & $20.3^{* *}$ & $20.30^{* *}$ \\
\hline BARI $\times$ DBL02 & $12.41 * *$ & 5.32 & 3.65 & $20.90^{* *}$ & -6.02 & $-14.99 *$ & 14.36 & $4.27 *$ & $14.65^{* *}$ & $-10.60^{*}$ & 14.43 & 14.44 \\
\hline SMB115 $\times$ Pant Samrat & $8.10^{* *}$ & $26.60 * *$ & $10.32^{* *}$ & $-27.76^{* *}$ & -3.84 & 6.18 & $38.95^{\text {** }}$ & $19.91^{* *}$ & $73.61^{* *}$ & 9.31 & $46.99 * *$ & $47.00^{* *}$ \\
\hline SMB115 $\times$ Pant Rituraj & $12.41 * *$ & $18.09^{* *}$ & -3.90 & $-46.87 * *$ & $76.76^{* *}$ & 4.98 & $40.11^{\text {** }}$ & $-28.57 * *$ & $32.41^{* *}$ & -2.67 & $38.33^{* *}$ & $38.33^{* *}$ \\
\hline SMB115 $\times$ DBL02 & 3.54 & $-14.89 *$ & -2.06 & $-10.73 * *$ & 11.58 & $64.50 * *$ & $122.80 * *$ & $25.17 * *$ & $95.76^{* *}$ & $55.53 * *$ & $116.52 * *$ & $116.59^{* *}$ \\
\hline BB85 $\times$ Pant Samrat & $-5.32 * *$ & 4.26 & -1.51 & $-28.82 * *$ & $17.81 * *$ & 42.66 ** & $35.34 * *$ & 14.46 ** & $29.35^{* * *}$ & $36.23 * *$ & $33.95^{* *}$ & $33.96^{* * *}$ \\
\hline BB85 $\times$ Pant Rituraj & $5.06^{* *}$ & -9.57 & 6.92 & $-43.39 * *$ & $87.62 * *$ & $-21.84 * *$ & $33.32 * *$ & $-10.51 * *$ & $54.37 * *$ & $-19.26^{* *}$ & $38.20^{* *}$ & $38.20^{* *}$ \\
\hline $\mathrm{BB} 85 \times$ DBL02 & $-5.57 * *$ & $13.83^{*}$ & 4.41 & $-15.80 * *$ & $18.66^{* *}$ & $15.92 *$ & $37.48^{* *}$ & -1.76 & $56.44 * *$ & $11.88^{*}$ & $41.87 * *$ & $41.86^{* *}$ \\
\hline BRLVAR6 $\times$ Pant Samrat & $15.44 * *$ & $56.38^{* *}$ & $8.81 *$ & $-15.27 * *$ & $26.21 * *$ & 3.57 & $50.29 * *$ & $-18.82 * *$ & $43.22 * *$ & -1.53 & $48.65^{* *}$ & $48.63^{* *}$ \\
\hline BRLVAR6 $\times$ Pant Rituraj & $21.01 * *$ & $40.43 * *$ & $16.49^{* *}$ & $-28.33^{* *}$ & $98.85^{* *}$ & $-15.40^{*}$ & $92.64 * *$ & $-21.01 * *$ & $109.08^{* *}$ & $-16.68 * *$ & $96.45^{* *}$ & $96.47^{* *}$ \\
\hline BRLVAR6 $\times$ DBL02 & 2.78 & $34.04 * *$ & 6.46 & $-26.44 * *$ & $-12.30^{*}$ & $-21.14 * *$ & $23.54 *$ & $-13.56 * *$ & $41.79^{* *}$ & $-19.42 * *$ & $27.77 * *$ & $27.79^{* *}$ \\
\hline IBWL2001-1 × Pant Samrat & $-4.56^{*}$ & -3.19 & $8.60 *$ & $-22.15^{* *}$ & $-12.33^{*}$ & 11.99 & $28.15^{* *}$ & $21.59^{* *}$ & $41.79 * *$ & $14.18^{* *}$ & $31.31 * *$ & $31.32 * *$ \\
\hline IBWL2001-1 × Pant Rituraj & $4.05^{*}$ & $-18.09 * *$ & 3.90 & $-20.37 * *$ & $89.62 * *$ & -5.75 & $84.26^{* *}$ & $-15.24 * *$ & $158.51^{* *}$ & -7.91 & $101.49^{* *}$ & $101.49 * *$ \\
\hline IBWL2001-1 × DBL02 & -1.52 & -6.38 & -3.11 & 4.26 & -6.58 & $-23.40 * *$ & 4.31 & -2.86 & $52.89^{* *}$ & $-18.72 * *$ & $15.57^{*}$ & $15.57^{*}$ \\
\hline PB66 $\times$ Pant Samrat & -2.78 & $28.72 * *$ & 1.30 & -3.13 & 9.70 & $91.43 * *$ & $104.83 * *$ & $92.01 * *$ & $128.32 * *$ & $91.56^{* * *}$ & $110.28 * *$ & $110.29 * *$ \\
\hline PB66 $\times$ Pant Rituraj & 2.53 & 1.06 & 0.76 & $-15.60^{* *}$ & $58.22 * *$ & 3.56 & $64.00^{* *}$ & $14.11^{* *}$ & $84.71^{* *}$ & 5.96 & $68.80^{* *}$ & $68.81 * *$ \\
\hline PB66 $\times$ DBL02 & $-4.05^{*}$ & $14.89^{*}$ & -6.25 & $-12.24 * *$ & $33.78 * *$ & $-25.07 * *$ & $56.55^{* *}$ & $-8.08^{* *}$ & $122.00^{* * *}$ & $-21.19 * *$ & $71.73^{* *}$ & $71.74^{* *}$ \\
\hline LC7 $\times$ Pant Samrat & $8.10 * *$ & $24.47 * *$ & $-7.72 *$ & $-20.23^{* *}$ & $44.35 * *$ & $-16.22 *$ & $59.07 * *$ & $-43.29 * *$ & $17.71^{* *}$ & $-22.40 * *$ & $49.47 * *$ & $49.46^{* *}$ \\
\hline LC7 $\times$ Pant Rituraj & $18.48^{* *}$ & -7.45 & -0.92 & $-40.26 * *$ & $89.61 * *$ & $-32.99 * *$ & $75.31^{* * *}$ & $-48.43 * *$ & $53.03^{* *}$ & $-36.51 * *$ & $70.14^{* *}$ & 70.14 ** \\
\hline LC7 $\times$ DBL02 & $9.62 * *$ & $20.21^{* *}$ & -1.26 & $-11.81^{* *}$ & $40.94 * *$ & $-47.91 * *$ & $49.53^{* *}$ & $-58.70 * *$ & $38.97 * *$ & $-50.37 * *$ & $47.08^{* *}$ & $47.05^{* *}$ \\
\hline PB70 $\times$ Pant Samrat & $4.81^{*}$ & $14.89 *$ & $8.69 *$ & -0.69 & $18.68 * *$ & $-37.76 * *$ & 17.73 & $-51.60 * *$ & -2.91 & $-40.92 * *$ & 12.94 & 12.96 \\
\hline PB70× Pant Rituraj & $18.23 * *$ & $30.85^{* *}$ & $16.50^{* *}$ & $-36.71 * *$ & $105.44 * *$ & $-53.17 * *$ & $54.37^{* * *}$ & $-43.52 * *$ & $115.29^{* *}$ & $-50.97 * *$ & $68.51^{* *}$ & $68.52^{* *}$ \\
\hline PB70 $\times$ DBL02 & 1.01 & $70.21^{* *}$ & 2.27 & $-18.22 * *$ & $52.05 * *$ & $-50.51 * *$ & 14.59 & $-59.77 * *$ & $25.70^{* *}$ & $-52.62 * *$ & $17.17^{*}$ & $17.16^{*}$ \\
\hline
\end{tabular}


Table.5 The best significant hybrids for different traits with respect to heterosis over mid parent, better parent and check variety

\begin{tabular}{|c|c|c|c|}
\hline Character & Heterosis over mid parent & Heterosis over better parent & Heterosis over standard check \\
\hline Days to $50 \%$ flowering & $\begin{array}{l}\text { BB85 } \times \text { Pant Samrat }(-7.43 \%) \\
\text { IBWL2001-1 } \quad \times \text { Pant Samrat } \\
6.80 \%) \\
\text { BRLVAR6 } \times \text { DBL02 }(-6.45 \%)\end{array}$ & $\begin{array}{l}\text { BRLVAR6 ×DBL02 }(-19.44 \%) \\
\text { PB70× DBL02 }(-14.93 \%) \\
\text { PB70 × Pant Samrat(-11.73\%) }\end{array}$ & $\begin{array}{l}\text { PB71 } \times \text { DBL02 }(-7.85 \%) \\
\text { BB85 } \times \text { DBL02 }(-5.57 \%) \\
\text { BB85 } \times \text { Pant Samrat }(-5.32 \%)\end{array}$ \\
\hline No. of primary branches plant ${ }^{-1}$ & $\begin{array}{l}\text { BRLVAR6× Pant Samrat }(81.48 \%) \\
\text { PB71 } \times \text { Pant Samrat }(65.00 \%) \\
\text { PB70 } \times \text { DBL02 }(53.85 \%)\end{array}$ & $\begin{array}{l}\text { BRLVAR6 } \times \text { Pant } \text { Samrat } \\
(56.38 \%) \\
\text { PB 70 × DBL02 }(49.53 \%) \\
\text { PB71 } \times \text { Pant Samrat }(40.43 \%)\end{array}$ & $\begin{array}{l}\text { PB70 } \times \text { DBL02 }(70.21 \%) \\
\text { BRLVAR6 } \times \text { Pant Samrat } \\
(56.38 \%) \\
\text { PB71 } \times \text { Pant Samrat }(40.43 \%)\end{array}$ \\
\hline Plant height $(\mathrm{cm})$ & $\begin{array}{l}\text { Swarn Syamli } \times \text { Pant Rituraj } \\
(23.22 \%) \\
\text { BB85 } \times \text { DBL02 }(22.74 \%) \\
\text { BRLVAR6 } \times \text { Pant Rituraj }(22.13 \%)\end{array}$ & 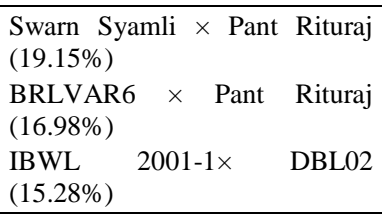 & $\begin{array}{l}\text { PB70 } \times \text { Pant Samrat }(16.50 \%) \\
\text { BRLVAR6 } \times \text { Pant Rituraj }(16 . \\
49 \%) \\
\text { PB } 71 \times \text { Pant Samrat }(16.37 \%)\end{array}$ \\
\hline Fruit length $(\mathrm{cm})$ & $\begin{array}{l}\text { Swarn Syamli } \times \text { Pant } \\
(47.25 \%)\end{array}$ & $\begin{array}{l}\text { Swarn Syamli } \times \text { Pant } \\
(34.95 \%) \\
\text { IBWL2001-1 } \times \text { Pant }\end{array}$ & BARI $\times$ DBL02 (20.90\%) \\
\hline Fruit diameter $(\mathrm{mm})$ & $\begin{array}{l}\text { PB66 × DBL02 }(22.80 \%) \\
\text { BARI } \times \text { Pant Samrat }(19.31 \%) \\
\text { BB85 } \times \text { Pant Rituraj }(18.97 \%)\end{array}$ & BARI $\times$ Pant Samrat $(15.44 \%)$ & $\begin{array}{l}\text { Sawarn Syamli } \times \text { Pant Rituraj } \\
(115.38 \%) \\
\text { PB70 × Pant Rituraj }(105.44 \%) \\
\text { BRLVAR6 } \times \text { Pant RIturaj } \\
(95.85 \%)\end{array}$ \\
\hline No. of marketable fruits plant ${ }^{-1}$ & $\begin{array}{l}\text { Swarn Syamli } \times \text { Pant } \\
(108.90 \%) \\
\text { Swarn Syamli } \times \text { Pant } \\
(108.76 \%) \\
\text { SMB115 } \times \text { DBL } 02(84.59 \%)\end{array}$ & $\begin{array}{l}\text { Swarn Syamli } \times \text { Pant Rituraj } \\
(97.94 \%) \\
\text { SMB115 } \times \text { DBL02 }(70.34 \%) \\
\text { PB66 } \times \text { Pant Samrat }(65.63 \%)\end{array}$ & $\begin{array}{l}\text { PB66 } \times \text { Pant Samrat }(91.43 \%) \\
\text { SMB115 } \times \text { DBL02 }(64.50 \%) \\
\text { Swarn Syamli } \times \text { Pant Samrat } \\
(47.42 \%)\end{array}$ \\
\hline Wt. of marketable fruits plant ${ }^{-1}$ & $\begin{array}{llll}\text { BRLVAR6 } & \times & \text { Pant } & \text { Rituraj } \\
(144.89 \%) & & & \\
\text { IBWL2001-1 } & \times & \text { Pant } & \text { Rituraj } \\
(130.84 \%) & & & \\
\text { SMB115 } \times \text { DBL02 } & (104.10 \%)\end{array}$ & $\begin{array}{l}\text { BRLVAR6 } \times \text { Pant Rituraj } \\
(135.78 \%) \\
\text { IBWL2001-1 } \times \text { Pant } \text { Rituraj } \\
(119.29 \%) \\
\text { BB85 × Pant Rituraj }(76.30 \%) \\
\end{array}$ & $\begin{array}{l}\text { SMB115 } \times \text { DBL02 }(122.80 \%) \\
\text { PB66 } \times \text { Pant Samrat }(104.83 \%) \\
\text { BRLVAR6 } \times \text { Pant Rituraj } \\
(92.64 \%)\end{array}$ \\
\hline No. of unmarketable fruits plant ${ }^{-1}$ & $\begin{array}{l}\text { PB70 } \times \text { DBL02 }(-51.41 \%) \\
\text { PB70 } \times \text { Pant Samrat }(-47.45 \%) \\
\text { Swarn Syamli } \times \text { Pant Samrat }(- \\
34.28 \%)\end{array}$ & $\begin{array}{l}\text { Swarn Syamli } \times \text { Pant Samrat }(- \\
52.41 \%) \\
\text { PB70 } \times \text { DBL02 }(-52.23 \%) \\
\text { PB70 } \times \text { Pant Samrat }(-51.60 \%)\end{array}$ & $\begin{array}{l}\text { PB70 } \times \text { DBL02 }(-59.77 \%) \\
\text { LC7 } \times \text { DBL02 }(-58.70 \%) \\
\text { Swarn Syami } \times \text { Pant Samrat }(- \\
52.41 \%)\end{array}$ \\
\hline Wt. of unmarketable fruits plant ${ }^{-1}$ & $\begin{array}{l}\text { Swarn Syamli } \times \text { Pant Samrat }(- \\
69.56 \%) \\
\text { PB70 } \times \text { Pant Samrat }(-39.79 \%) \\
\text { PB70 } \times \text { DBL02 }(18.81 \%)\end{array}$ & $\begin{array}{l}\text { Swarn Syamli } \times \text { Pant Samrat }(- \\
63.69 \%) \\
\text { PB } 70 \times \text { Pant Samrat }(-56.36 \%) \\
\text { PB70 } \times \text { DBL02 }(-43.50 \%)\end{array}$ & $\begin{array}{l}\text { Swarn Syamli } \times \text { Pant Samrat }(- \\
63.67 \%)\end{array}$ \\
\hline Total no. of fruits plant ${ }^{-1}$ & $\begin{array}{l}\begin{array}{l}\text { Swarn Syamli } \times \text { Pant } \\
(83.33 \%)\end{array} \\
\text { SMB115 } \times \text { DBL02 }(77.08 \%) \\
\text { Swarn Syamli } \times \text { Pant Samrat } \\
(75.58 \%)\end{array}$ & $\begin{array}{l}\text { Swarn Syamli } \times \text { Pant Rituraj } \\
(67.99 \%) \\
\text { SMB115 } \times \text { DBL02 }(65.73 \%) \\
\begin{array}{l}\text { BRLVAR6 } \times \text { Pant Rituraj } \\
(63.02 \%)\end{array}\end{array}$ & $\begin{array}{l}\text { PB66 } \times \text { Pant Samrat }(91.56 \%) \\
\text { SMB115 } \times \text { DBL02 }(55.53 \%) \\
\text { BB85 } \times \text { Pant Samrat }(36.23 \%)\end{array}$ \\
\hline Total yield plant ${ }^{-1}(\mathrm{~kg})$ & $\begin{array}{llcl}\begin{array}{l}\text { IBWL2001-1 } \\
(159.31 \%)\end{array} & \times & \text { Pant } & \text { Rituraj } \\
\text { BRLVAR6 } & \times & \text { Pant } & \text { Rituraj } \\
(147.54 \%) & & & \\
\text { SMB115 } \times \text { DBL02 } & (95.16 \%) & \end{array}$ & $\begin{array}{l}\text { IBWL2001-1 } \times \text { Pant } \text { Rituraj } \\
(157.59 \%) \\
\text { BRLVAR6 } \times \text { Pant } \quad \text { Rituraj } \\
(144.04 \%) \\
\text { BB85 } \times \text { Pant Rituraj }(76.68 \%)\end{array}$ & $\begin{array}{l}\text { SMB115 × DBL02 }(116.52 \%) \\
\text { PB66 × Pant Samrat }(110.28 \%) \\
\text { IBWL2001-1× Pant Rituraj } \\
(101.49 \%)\end{array}$ \\
\hline Total yield ha ${ }^{-1}$ (q) & $\begin{array}{llll}\begin{array}{l}\text { IBWL2001-1 } \\
(159.30 \%)\end{array} & \times & \text { Pant } & \text { Rituraj } \\
\text { BRLVAR6 } & \times & \text { Pant } & \text { Rituraj } \\
(144.05 \%) & & & \\
\text { SMB115 } \times \text { DBL02 } & (95.22 \%) & \\
\end{array}$ & $\begin{array}{l}\text { IBWL2001-1 } \times \text { Pant } \text { Rituraj } \\
(157.67 \%) \\
\text { BRLVAR6 } \times \text { Pant } \quad \text { Rituraj } \\
(144.05 \%) \\
\text { BB85 } \times \text { Pant Rituraj }(76.74 \%) \\
\end{array}$ & $\begin{array}{l}\text { SMB115 × DBL02 }(116.59 \%) \\
\text { PB66 × Pant Samrat }(110.29 \%) \\
\text { IBWL2001-1× Pant Rituraj } \\
(101.49 \%)\end{array}$ \\
\hline
\end{tabular}


Fruit length exhibited positive significant relative heterosis, heterobeltiosis and economic heterosis 13, 2 and 1 cross, respectively. Maximum positive relative heterosis was recorded in cross Swarn Syamli $\times$ Pant Rituraj (47.25\%), again maximum heterobetiosis was shown by the cross Swarn Syamli $\times$ Pant Rituraj (34.95\%) and maximum economic heterosis was observed in BARI $\times$ DBL02 (20.90\%). Similar results were reported by Ansari et al., (2009), Das et al., (2009), Biswas et al., (2013) and Kumar et al., (2013). Fruit diameter exhibited positive significant relative heterosis, heterobeltiosis and economic heterosis with 6 , 1 and 22 crosses, respectively. Maximum positive relative heterosis was recorded in cross PB66 $\times$ DBL02 $(22.80 \%)$, maximum heterobeltiosis in BARI $\times$ Pant Samrat $(15.44 \%)$ and maximum economic heterosis was recorded in Sawarn Syamli $\times$ Pant Rituraj $(115.38 \%)$.The result was in agreement with earlier studies Ashwani and Khandelwal (2003) and Das et al., (2009).

Results revealed that 21, 11 and 6 crosses showed significant positive relative heterosis, heterobeltiosis and standard heterosis, respectively. Highest magnitude of relative heterosis, heterobeltiosis and standard heterosis was recorded in crosses Swarn Syamli $\times$ Pant Samrat (108.90\%), Swarn Syamli $\times$ Pant Rituraj (97.94\%) and PB66 $\times$ Pant Samrat (91.43\%), respectively. Ansari et al., (2009) and Biswas et al., (2013) quoted similar results.

For weight of marketable fruits per plant 25, 20 and 22 crosses exhibited positive relative heterosis, heterobeltiosis and economic heterosis, respectively. Highest magnitude of relative heterosis, heterobeltiosis and standard heterosis was recorded in crosses BRLVAR6 $\times$ Pant Rituraj (144.89\%), BRLVAR6 × Pant Rituraj $(135.78 \%)$ and SMB115 $\times$ DBL02 $(122.80 \%)$ respectively. Similar result was reported by Biswas (2013).
For number of unmarketable fruits per plant negative heteorsis is desirable and 9, 14 and 20 crosses exhibited significant negative relative heterosis, heteorbeltiosis and economic heterosis respectively. Highest negative magnitude of relative heterosis, heterobeltiosis and standard heterosis was recorded in crosses PB70 $\times$ DBL02 ($51.41 \%)$, Swarn Syamli $\times$ Pant Samrat $(-$ $52.41 \%)$ and PB70 $\times$ DBL02 (-59.77\%) respectively. These results are in agreement with the findings of Kumar and Pathania (2003).

For weight of unmarketable fruits per plant also negative heterosis is desirable and 3, 6 and 1 cross combination showed significant negative relative heterosis, heteorbeltiosis and economic heterosis, respectively. Highest negative magnitude of relative heterosis, heterobeltiosis and standard heterosis was recorded in cross Swarn Syamli $\times$ Pant Samrat $(-9.56 \%)$, Swarn Syamli $\times$ Pant Samrat $(-63.69 \%)$ and Swarn Syamli $\times$ Pant Samrat (-63.67\%) respectively.

These observations were in close agreements with Kumar and Pathania (2003).

Total number of fruits plant $^{-1}$ exhibited positive significant relative heterosis, heterobeltiosis and economic heterosis with 22, 12 and 5 crosses, respectively. Maximum positive relative heterosis was recorded in cross Swarn Syamli $\times$ Pant Rituraj (83.33\%), maximum positive heterobeltiosis was again showed by cross Swarn Syamli $\times$ Pant Rituraj $(67.99 \%)$ and maximum positive economic heterosis was exhibited by cross PB66 $\times$ Pant Samrat $(91.56 \%)$. Ashwani and Khandelwal (2003) and Biswas et al., (2013) reported similar results. Yield in any crop is the final product of different yield components. This ultimate produce in the plant is expressed through mutual balancing of characters. Total yield plant $^{-1}$ exhibited positive significant relative heterosis, heterobeltiosis and 
economic heterosis with 24, 22 and 25 crosses, respectively. Maximum positive relative heterosis was recorded in cross IBWL2001-1 × Pant Rituraj (159.31\%), maximum positive heterobeltiosis in again same cross IBWL2001-1 × Pant Rituraj (157.59\%) and maximum positive economic heterosis was shown by cross SMB $115 \times$ DBL02 (116.52\%). These findings are in agreement with the results of Ansari et al., (2009) and Biswas et al., (2013). For number of marketable fruits per plant and total number of fruits per plant, cross PB66 $\times$ Pant Samrat has high per se performance as well as it showed maximum economic heterosis for above traits. Whereas for weight of marketable fruits per plant, total yield per plant and yield per hectare cross, SMB115 $\times$ DBL02 has high per se performance and maximum economic heterosis for above mentioned traits.

\section{References}

Ansari AM, Ahmad E, Sah A, Ali MN and Bhagat BK, 2009. Studies of Heterosis in Brinjal. Green Farming 2 (2): 211-214.

Aswani RC and Khandelwal RC, 2005. Combining ability studies in brinjal. Indian Journal of Horticulture 62(1): 37-40.

Biswas L, Mehta N and Ansari SF, 2013. Hybrid Vigour Studies in Brinjal (Solanum Melongena L.). Global Journal of Science Frontier Research Agriculture and Veterinary 13(9): 9-11.

Das S, Mandal AB and Hazra P, 2009. Study of heterosis in brinjal (Solanum melongena $\mathrm{L}$.) for yield attributing traits. Journal of Crop and Weed 5(2): 25-30.

Dubey R, Das A, Ojha MD, Saha B, Ranjan A and Singh PK, 2014. Heterosis and combining ability studies for yield and yield attributing traits in brinjal (Solanum melongena L.). The Bioscan 9(2): 889-894.

Hedges LJ and Lister CE, 2007. Nutritional attributes of spinach, silver beet and eggplant. Crop Food Res Confidential Rep No. 1928.

Kempthorne D, 1957. An introduction to genetic statistics. John Wiley \& Sons. New York, pp. $468-471$.

Kumar SR, Arumugam T, Anandakumar CR and Rajavel, DS 2013. Outbreeding for yield and horticultural attributes in indigenous eggplant germplasm. African Journal of Agricultural Research 8(29): 4099-4110.

Kumar V and Pathania NK, 2004. Combining ability studies in brinjal (Solanum melongena L.). Vegetable Science 30(1): 50-53.

Mariola P, Isabel A, Santiago V, Maria H, Pietro G, Francisco JH and Jaime P, 2013. Breeding for chlorogenic acid content in eggplant: interest and prospects. Notulae Botanicae Horti Agrobotanici Cluj-Napoca 41(1): 26-35

Ojiewo, C.O., Murakami, K., Masinde, P.W. and Agong, S.J. 2007. Mutation breeding of african nightshade (Solanum section Solanum). Fruit Veg Cereal Biotech. 1. 3952.

Rai M, Gupta PN and Agarwal RC, 1995. Catalogue on eggplant (Solanum Melongena L.) germplasm Part - I. National Bureau of Plant Genetic Resources, Pusa Campus, New Delhi, pp. 1-3.

Singh HV, Singh SP, Singh M and Singh S, 2002. Genetic analysis of quantitative traits in brinjal (Solanum melongena L.). Vegetable Science 29(1): 84-86.

Tiwari A, Rajesh SJ, Piyush T and Nayak S, 2009. Phytochemical investigations of crown of Solanum melongena fruit. International Journal Phytomedicin. 1:9-11.

\section{How to cite this article:}

Pramila, M.L. Kushwaha and Yamuna Prasad Singh. 2017. Studies on Heterosis in Brinjal (Solanum melongena L.). Int.J.Curr.Microbiol.App.Sci. 6(11): 641-651. doi: https://doi.org/10.20546/ijcmas.2017.611.077 